\title{
Sociocultural Determinants of Teenage Childbearing Among Latinas in California
}

\author{
Christine Dehlendorf · Kristen Marchi • \\ Eric Vittinghoff · Paula Braveman
}

Published online: 27 January 2009

(C) The Author(s) 2009. This article is published with open access at Springerlink.com

\begin{abstract}
Objectives U.S. Latinas have a persistently high rate of teenage childbearing, which is associated with adverse outcomes for both mother and child. This study was designed to investigate the roles of socioeconomic factors and acculturation in teenage childbearing in this population. Methods Logistic regression was used to analyze the association of measures of acculturation (language spoken at home, nativity, and age at immigration) and respondents' parents' education with age at first birth in a stratified sample of post-partum women in California. Results The unadjusted odds ratio for teenage birth for Latinas versus non-Latina Whites was 5.2 (95\% CI 4.16.6). Nativity was not significantly associated with teen birth, but speaking Spanish at home was positively associated and immigrating at a later age was negatively associated with teen birth. Overall, these measures of acculturation accounted for $17 \%$ (95\% CI 8-28\%) of the difference in odds of teen birth between Latinas and nonLatina Whites. Higher levels of education among respondents' parents had differentially protective effects across the racial/ethnic groups. Controlling for disparities in respondents' parents' education without changing its differential effects across racial/ethnic groups reduced the odds ratio for Latinas compared to non-Latina Whites by $30 \%$ (95\% CI 14-60\%). Conclusion These findings call
\end{abstract}

C. Dehlendorf $(\varangle) \cdot$ K. Marchi · P. Braveman

Department of Family and Community Medicine, University of California San Francisco, 995 Potrero Avenue, Ward 83, San Francisco, CA 94110, USA

e-mail: cdehlendorf@fcm.ucsf.edu

E. Vittinghoff

Department of Epidemiology and Biostatistics, University of California San Francisco, 185 Berry Street, Lobby 5, Suite 5700, San Francisco, CA 94107, USA into question common assumptions about the protective effect of acculturation on teen fertility and suggest that improving childhood socioeconomic factors among Latinas may decrease teen childbearing.

Keywords Latinas - Socioeconomic status · Socioeconomic position - Acculturation . Adolescent health $\cdot$ Teen pregnancy

\section{Introduction}

Births to adolescent mothers are associated with adverse outcomes, including pregnancy complications, [1] lower educational attainment, [2] and increased rates of subsequent poverty $[3,4]$ for the mother and increased risk of neglect, [5] behavioral problems, [6] and low educational achievement [7] for the child. Nationally, Latina adolescents have a higher birth rate than non-Latina Whites with a rate of 81 per 1,000 , compared to 26 per 1,000 among non-Latina White adolescents [8]. Rates of adolescent childbirth have been decreasing overall in the United States, but have decreased more slowly among Latinas. Between 1991 and 2005, teen birth rates among both nonLatina Whites and African Americans declined more than $50 \%$ and $48 \%$, respectively, yet the rate among Latinas declined by only $22 \%$ [8]. Decreasing the rate of teen pregnancy among Latinas, and eliminating the disparities between Latinas and non-Latina Whites, is recognized as a priority by the public health community, including the Centers for Disease Control and Prevention [9] and the National Campaign to Prevent Teen Pregnancy [10]. In order to accomplish these goals, more knowledge is needed about the causes of the persistently high childbearing rate among Latina adolescents. 
Previous research has identified differences in sexual activity, contraceptive use, and access to health services as possible contributors to differences in adolescent pregnancy and childbirth by race/ethnicity $[11,12]$. Additional research has examined distal influences on these behaviors. Specifically, measures of exposure and adaptation to U.S. culture ("acculturation") such as birthplace, number of years spent in the U.S., and language have frequently been studied as predictors of reproductive health outcomes among Latina adolescents [13].

One commonly mentioned hypothesis is that less acculturated teens have higher levels of teenage childbearing because they are more affected by value systems from their countries of origin that support early fertility [14-16]. This theory is not well supported by current evidence. In fact, measures of greater acculturation have been associated with increased levels of sexual activity, including earlier age at sexual initiation and greater number of lifetime partners, while the relationship between measures of acculturation and contraception use has been inconsistent [13, 17]. Further, teen fertility rates have rarely been examined in studies of acculturation, with one study finding a relationship between preferring to communicate in English and higher adolescent pregnancy rates, and no studies investigating teen birth rates [13]. Recent demographic evidence from Mexico also is inconsistent with this theory, since, as of 1998, teenage fertility rates in Mexico have been lower than the teenage fertility rates of women of Mexican origin living in the United States [15]. More generally, the measurement of acculturation as a predictor of health outcomes such as teen childbearing has been questioned due to the challenges encountered in defining and measuring the complex concepts of culture and cultural change [18].

In comparison to measures of acculturation, childhood socioeconomic factors as predictors of teenage reproductive health outcomes among Latinas have received relatively little attention, [19] despite the fact that lower socioeconomic status or position has been shown to be associated with earlier initiation of sexual intercourse and with adolescent pregnancy and childbirth among Blacks and Whites [20-27] and that Latinas in the United States in general have lower levels of education [28] and other socioeconomic indicators [29] than non-Latina Whites. We identified a few studies that investigated the association of socioeconomic factors with precursors to teen childbearing among Latinas. One study found a positive relationship between maternal education and the perceived importance of birth control, [30] and another found a positive association between receipt of welfare and teen pregnancy [31]. With respect to teen childbearing, we could identify only one study that investigated the effect of socioeconomic status in this population. Using data from 1994, Driscoll et al. [32] investigated the independent effects of community opportunity, family socioeconomic factors, and educational expectations on teen childbirth and did not find an effect of family socioeconomic status in Latinas, while there was an association for both Whites and African Americans.

To further elucidate the relationships between acculturation and childhood socioeconomic factors and Latina adolescent reproductive health, this study used data from a statewide California survey of post-partum mothers to investigate the associations of language, nativity, years in the United States, and the educational attainment of respondents' parents with teen birth among first time Latina mothers. This study was approved by the University of California, San Francisco Committee on Human Research and by the State of California's Committee for the Protection of Human Subjects.

\section{Methods}

\section{Data Source}

Data from primiparous Latinas and non-Latina Whites completing the Maternal and Infant Health Assessment (MIHA) survey from 2003 through 2005 were used in these analyses. The MIHA survey is a collaborative effort of the California Department of Public Health, Maternal, Child and Adolescent Health Program, and researchers at the University of California, San Francisco. It is an annual survey of mothers delivering in California between February and May, linked with birth certificate data. Women are eligible if they speak English or Spanish, are 15 years old or older, and have addresses available on the birth certificate of the index pregnancy. Potential participants are randomly selected from birth certificate data stratified by region in California, education, and race. The survey is sent by mail 10-14 weeks after the index birth and is selfadministered. Reminder postcards and second mailings of the questionnaire are sent to non-responders. Telephone interviews are attempted for all non-responders to the mail survey, and the overall response rate has been approximately $70 \%$ or greater each year.

\section{Sample}

The MIHA sample from 2003 to 2005 consisted of 3,103 primiparous Latina or non-Latina White women who spoke English or Spanish. The 555 Latina mothers who began living in the United States after age 14 were excluded from the sample in order to prevent biased sampling of immigrant women. The 153 non-Latina White women who did not speak English or were not born in the United States were also excluded. An additional 276 women of both ethnicities were excluded for missing data. The sample was weighted 
to adjust for the stratified sampling design and nonresponse. The final sample size, after exclusions, was 2,119 .

\section{Variables}

All variables were obtained from MIHA data unless otherwise indicated.

\section{Teen Birth}

Age at first birth was determined by the age of the mother recorded on the birth certificate and categorized as aged 15-19 years or aged 20 or older.

\section{Race/Ethnicity}

- In the MIHA survey, women were asked to choose their race/ethnicity from a list of six options (African American, American Indian, Asian/Pacific Islander, Latina, White, or Other). If they chose more than one option, they were asked to select the one with which they most identified. Women were then categorized as non-Latina White or Latina for this study.

\section{Measures of Acculturation}

- Language was defined as what language the woman reported usually speaking at home at the time of the interview (English, Spanish, or English and Spanish equally).

- The nativity of the respondent was self-reported and categorized as "US-born" or "non-US-born."

- Age at which the woman immigrated to the United States was a continuous variable and calculated by subtracting the woman's birth year (as recorded in the birth certificate) from the year she reported coming to the United States. Women born in the United States were assigned a value of zero. We did not use the number of years living in the United States as a variable because our sample consisted of woman of varying ages, and our question of interest was regarding the effect of the number of years they had lived in the United States prior to age 15, when they became at risk of teen childbearing as defined in our analysis.

\section{Respondents' parents' educational attainment ("Parental education”) as a measure of respondents' childhood socioeconomic status}

The highest level of education of either of the respondent's parents when she was 13 years old was used as a measure of childhood socioeconomic status. The respondent's highest level of parental education was determined by the response to the question: "Thinking back to who you lived with when you were about 13 years old, what was the highest grade or year of school completed by your mother, father, or main guardian?" It was categorized as "did not finish high school," "high school graduate/GED," "some college," or "college graduate."

Analyses

Descriptive analyses were performed using chi-squared tests for categorical variables and $t$ tests for continuous variables.

We used logistic regression to investigate the association of our measures of acculturation with teen birth. We examined the extent to which overall disparities in teen childbearing by race/ethnicity were mediated by measures of acculturation by determining percent changes in the unadjusted log odds-ratios (ORs) for teen childbearing, contrasting Latinas with non-Latina Whites, after adjustment for language alone and place of birth and age at immigration alone, and then combining all acculturation measures. Bootstrap methods were used to compute biascorrected percentile confidence intervals for the percent changes in the log ORs. We verified that the continuous variable of age at which the woman began living in the United States did not violate the log linearity assumption when entered into the model with place of birth.

To study the association of respondents' highest level of parental education with teen childbirth, we performed logistic regression analyses stratified by race/ethnicity to determine the effect of parental education within each racial/ethnic group. As a significant interaction was identified between respondents' parental education and race/ ethnicity, we were unable to incorporate parental education and measures of acculturation into the same model. Therefore, we could not calculate the percentage reduction in odds of teen childbearing for parental education in the same manner as for measures of acculturation. We therefore estimated the potential effect of increasing parental education among US-born and non-US-born Latinas to the levels observed among non-Latina Whites, without modifying its differential effect across groups, using a counterfactual sample for each Latina subgroup. In each sample, the distribution of parental education was manipulated to match that of the non-Latina Whites, but the effects of parental education were computed using separate estimates for US-born and immigrant Latinas, respectively, based on a logistic model including the appropriate interactions and fit to the actual observations. We then computed the $\log$ OR for each of the two counterfactual Latina groups with respect to non-Latina Whites and assessed the percent changes in the log ORs attributable to 
adjustment for parental education, again with bias-corrected percentile confidence intervals computed using bootstrap methods. The results are interpretable as the potential effect of overcoming disparities in respondents' parental education, without changing the differential effects of this factor across the three groups.

The survey logistic function in Stata Version 9.2 (Stata Corp, College Station, TX) was used to estimate all logistic models, taking account of the stratification of the sample as well as sampling weights. $P$ values $<0.05$ were considered statistically significant.

\section{Results}

Characteristics of the sample of primiparous Latina and non-Latina White women in California are presented in Table 1. Non-Latina White women and women whose parents had higher levels of education were less likely to have a first birth before the age of 20 . No marked differences in age at first birth were seen relative to place of birth or the age at which the subject began living in the United States among Latina mothers. Latina teen mothers were more likely to speak Spanish at home, whether or not they also spoke English. The majority $(83.1 \%)$ of immigrant Latinas in this study were from Mexico, which mirrors the California population (data not shown).
Measures of Acculturation

Table 2 presents the results of a logistic regression model for teen birth including race/ethnicity and the three measures of acculturation. Latina women had a 5.2 (95\% CI 4.1-6.6) higher odds of having a teen birth compared to non-Latina White women, which decreased to 3.8 (95\% CI 2.9-5.1) when language was incorporated into the model. No further attenuation was observed when place of birth and age the mother began living in the US were also taken into consideration. Among immigrant Latinas, there was a slightly significant decrease in the odds of teen birth associated with older age at immigration (OR 0.93 for every year of age, 95\% CI 0.88-0.99). In total, when controlling for measures of acculturation, the odds of teen birth decreased from 5.2 to 3.9 , an attenuation of $17 \%$ (95\% CI 8-28\%).

In the fully adjusted model, the estimated effects of language were large among subgroups. Specifically, the odds ratio for having a teen birth among US-born Latinas who spoke Spanish at home was 9.2 (95\% CI 6.3-13.5) compared to non-Latina Whites, while US-born Englishspeaking Latinas had an OR of 3.9 (95\% CI 2.9-5.2). Among immigrants, age at immigration had a strong effect on whether a woman had a teen birth. Immigrant Latinas who moved to the US prior to the age of one and reported speaking English at home had an OR of 4.5 (96\% CI 2.5-8.1) compared to non-Latina Whites, while those who

Table 1 Characteristics of first time mothers in the California Maternal and Infant Health Assessment Survey, 2003-2005

\begin{tabular}{|c|c|c|c|}
\hline All mothers & $\begin{array}{l}\text { First birth age } 15-19 \\
N=441(\%)\end{array}$ & $\begin{array}{l}\text { First birth age } 20 \text { or older } \\
N=1678(\%)\end{array}$ & $\begin{array}{l}P \text { value for comparison } \\
\text { of first birth }<20 \text { and } \geq 20\end{array}$ \\
\hline \multicolumn{4}{|l|}{ Race/ethnicity } \\
\hline Non-Latina White & 28.2 & 67.2 & \multirow[t]{2}{*}{$<0.001$} \\
\hline Latina & 71.8 & 32.8 & \\
\hline \multicolumn{4}{|c|}{ Respondent's highest parental education } \\
\hline Did not finish high school & 46.3 & 18.3 & \multirow[t]{4}{*}{$<0.001$} \\
\hline High school graduate/GED & 25.7 & 21.5 & \\
\hline Some college & 20.4 & 25.2 & \\
\hline College graduate or more & 7.6 & 35.0 & \\
\hline Latina mothers only & $N=315(\%)$ & $N=555(\%)$ & \\
\hline \multicolumn{4}{|l|}{ Nativity } \\
\hline US-born & 70.9 & 70.3 & \multirow[t]{2}{*}{0.85} \\
\hline Non-US-born & 29.1 & 29.7 & \\
\hline \multicolumn{4}{|c|}{ Age began living in US for immigrant Latinas } \\
\hline Mean (SD) & $6.7(4.6) n=90$ & $7.8(4.3) n=158$ & 0.11 \\
\hline \multicolumn{4}{|l|}{ Language spoken at home } \\
\hline English & 39.1 & 53.3 & \multirow[t]{3}{*}{$<0.001$} \\
\hline English and Spanish equally & 30.8 & 24.5 & \\
\hline Spanish & 30.0 & 22.2 & \\
\hline
\end{tabular}


Table 2 Odds of a teenage birth among Latinas, compared to non-Latina Whites, unadjusted and adjusted for measures of acculturation

\begin{tabular}{|c|c|c|c|c|}
\hline & $\begin{array}{l}\text { Unadjusted } \\
\mathrm{OR}^{\mathrm{a}}(95 \% \mathrm{CI})\end{array}$ & $\begin{array}{l}\text { OR adjusted } \\
\text { for language } \\
(95 \% \mathrm{CI})\end{array}$ & $\begin{array}{l}\text { OR adjusted for nativity } \\
\text { and age at immigration } \\
(95 \% \mathrm{CI})\end{array}$ & $\begin{array}{l}\text { OR adjusted for language, } \\
\text { nativity, and age at } \\
\text { immigration }(95 \% \mathrm{CI})\end{array}$ \\
\hline Latina (ref.: non-Latina White) & $5.2(4.1-6.6)$ & $3.8(2.9-5.1)$ & $5.3(4.1-6.7)$ & $3.9(2.9-5.2)$ \\
\hline \multicolumn{5}{|l|}{ Nativity and age at immigration } \\
\hline Non-US-born (ref.: US-born) & $3.5(2.1-5.9)$ & $-{ }^{\mathrm{b}}$ & $1.4(0.82-2.4)$ & $1.1(0.67-2.0)$ \\
\hline $\begin{array}{l}\text { Age began living in US } \\
\text { (Equal to } 0 \text { for US-born Latinas) }\end{array}$ & $0.95(0.90-1.0)$ & $-{ }^{b}$ & $0.95(0.90-1.0)$ & $0.93(0.88-0.99)$ \\
\hline \multicolumn{5}{|l|}{ Language spoken at home (ref.: English) } \\
\hline English and Spanish equally & $4.1(3.1-5.6)$ & $1.7(1.2-2.4)$ & $-{ }^{\mathrm{b}}$ & $1.9(1.3-2.6)$ \\
\hline Spanish & $4.4(3.3-6.0)$ & $1.8(1.3-2.6)$ & $-{ }^{\mathrm{b}}$ & $2.4(1.6-3.5)$ \\
\hline
\end{tabular}

a Calculated using single predictor logistic models

b Not included in model

spoke Spanish had an OR of 10.6 (95\% CI 5.8-19.3). These ORs decreased with each year increase in age at which the woman came to the US and were higher among those who moved at age 14 for women who still spoke Spanish at home [4.0 (95\% CI 2.4-6.8)] than for those who spoke English [1.7 (95\% CI 0.93-3.1)] (data not shown).

In a sensitivity analysis restricted to Latinas, controlling for parental education (which had similar effects in the US- and non-US-born) did not affect the estimated associations of teen birth with language and nativity of the respondent.

\section{Respondents’ Parents’ Education}

In preliminary analysis of the association of respondents' highest parental education with teen birth, a significant interaction with race/ethnicity was identified $(P=0.003)$. The stratified results by race/ethnicity are displayed in Table 3. Among non-Latina Whites, parents' educational attainment of high school graduate or beyond was associated with a significant decrease in odds of teen birth, while among Latinas, only having a college educated parent was significantly associated with a decreased risk. Overall, lower levels of respondents' parents' education were associated with higher odds of teen births in both nonLatina Whites $(P<0.001)$ and Latinas $(P=0.002)$. The association between parental education and odds of teen
Table 3 The odds of teen birth associated with respondent's highest level of parental education, stratified by race/ethnicity and adjusted for measures of acculturation

\begin{tabular}{lll}
\hline & Non-Latina Whites & Latinas \\
\hline $\begin{array}{l}\text { Respondent's highest parental education (ref.: did not finish high } \\
\text { school) }\end{array}$ & \\
High school graduate/GED & $0.32(0.16-0.64)$ & $0.97(0.67-1.4)$ \\
Some college & $0.23(0.12-0.45)$ & $0.95(0.62-1.4)$ \\
College graduate or more & $0.08(0.04-0.16)$ & $0.32(0.16-0.63)$ \\
\hline
\end{tabular}

Model includes respondent's highest level of parental education when she was 13, language spoken at home, nativity, and age at immigration

births was not significantly different between US-born and immigrant Latinas.

The effect of controlling for respondents' highest level of parental education on the odds of teen birth is shown in Table 4. In the actual sample, the OR for teen birth among US-born English-speaking Latinas with respect to nonLatina Whites was 3.9 (95\% CI 2.9-5.2). In the counterfactual sample, increasing the level of parental education to the levels observed among non-Latina Whites, while maintaining the race/ethnicity/nativity-specific effects of respondents' parents' education, decreased the OR to 2.8 (95\% CI $1.8-4.3$ ), a $23 \%$ decrease (95\% CI $2-52 \%)$. The

Table 4 Odds ratio for teen birth by race/ethnicity and place of birth in actual sample and in sample adjusted for respondent's highest level of parental education

\begin{tabular}{|c|c|c|c|}
\hline & US-born Latinas & Immigrant Latinas & All Latinas \\
\hline Odds ratio for teenage birth in actual sample (95\% CI) (ref.: non-Latina White) & $3.9(2.9-5.2)$ & $4.5(2.5-8.1)$ & $3.9(2.9-5.2)$ \\
\hline $\begin{array}{l}\text { Odds ratio for teen birth in sample adjusted for parental education } \\
(95 \% \text { CI) (ref.: non-Latina White) }\end{array}$ & $2.8(1.8-4.3)$ & $2.0(0.8-4.8)$ & $2.6(1.7-3.7)$ \\
\hline $\begin{array}{l}\text { Percent reduction in odds ratio after setting parental education } \\
\text { equal to that of non-Latina Whites }(95 \% \mathrm{CI})\end{array}$ & $23(2-52)$ & $55(17-111)$ & $30(14-60)$ \\
\hline
\end{tabular}

Models include respondent's highest level of parental education when she was 13, language spoken at home, nativity, and age at immigration 
corresponding reduction in the OR comparing immigrant Latinas to non-Latina Whites was from 4.5 (95\% CI $2.5-8.1)$ to 2.0 (95\% CI $0.8-4.8)$, a $55 \% \quad(95 \%$ CI $17-111 \%$ ) decrease. Combining both the US-born and immigrant Latinas, the percent reduction in excess odds of teen childbearing was $30 \%$ (95\% CI 14-60\%).

\section{Discussion}

In this population-based sample of primiparous women in California, language spoken at home and the mothers' parents' educational attainment were both significantly associated with teen childbearing. Speaking both English and Spanish or only Spanish at home accounted for $17 \%$ of the increase in the log-odds of teen birth among Latinas compared to non-Latina Whites, while parental education explained 23\% of the increase among US-born Latinas and $55 \%$ of the increase among immigrant Latinas. While outcomes that are precursors to teen birth, such as sexual activity and pregnancy, have been studied previously, this is, to our knowledge, the first study to examine measures of acculturation in relation to the outcome of teen birth among Latinas and the second study to investigate measures of socioeconomic status with respect to this outcome.

Our results differ from those of Driscoll et al. [32] whose previous study did not find an independent association of family socioeconomic factors on teen birth. This discrepancy could reflect temporal trends, as their data were collected over a decade before those used in the present study; it also may reflect the inclusion of community opportunity and educational expectations in the multivariable model in the Driscoll study, thereby potentially controlling for all or part of the pathway by which familial socioeconomic status or position affects teen birth.

Our findings call into question widespread assumptions regarding the nature of associations between some measures of acculturation and teenage birth. In contrast to the hypothesis that greater time spent living in the United States reduces teen birth rates among immigrants, we found the opposite pattern. Specifically, as compared to non-Latina Whites, teen birth rates were comparably elevated among Latinas born within the United States and those who immigrated very early in life; however, among Latinas born outside the United States, the odds of teen birth decreased significantly with each additional year of age at immigration (up until age 14, our study cut-off). Thus Latinas who immigrated at age 13 or 14 appeared to be at substantially lower risk for teen birth than both US-born Latinas and Latinas who immigrated in the first years of life. The effect of time spent in the United States does not appear to be confounded by language spoken at home, as these patterns were similar in models with and without control for language. While the causes of the negative association between increased time spent outside the United States and teen childbirth cannot be determined using our data, these findings are potentially consistent with the presence of protective cultural values, including religious beliefs and strong family ties, among Latinas with strong connections to their countries of origin $[10,33]$. Research on the effect of these cultural values on teen fertility could inform future interventions designed to decrease the rate of teen childbearing among Latinas.

In contrast to the effect of time spent living in the United States, however, the effect of language observed in this study is consistent with a protective effect of increasing acculturation, and was particularly evident among Latinas who were born in the United States but spoke Spanish at home, whose odds of teen birth were highest as compared to non-Latina Whites. This effect of language also may be related to access to care, the current educational level of the household or other factors unrelated to cultural norms associated with the immigrant's country of origin, indicating the need for additional research to further delineate these factors.

The influence of the respondents' highest level of parental education on teenage childbearing could operate via many different pathways. Living in a low-income neighborhood has been associated with a higher incidence of teen birth, [26, 32, 34] and may be one pathway through which familial disadvantage affects teen fertility. Parents with higher educational levels may communicate more with their daughters in general and/or specifically about sex and contraception [35]. Children raised by parents with higher levels of educational attainment may be instilled with higher levels of expectations about their own educational attainment and career, motivating them to avoid becoming teenage mothers, [36] and may have greater access to information about the educational system, [37] giving them a greater sense of opportunity. Parental education also may be a proxy for higher levels of income or wealth in a woman's childhood environment; higher income or wealth can facilitate greater family stability, [38] which may discourage teen childbearing.

Limitations of these analyses include the difficulty of measuring both acculturation and childhood socioeconomic circumstances. We used language spoken at home at the time of the interview, nativity, and age at which the woman began living the US as our measures of acculturation. While the use of multiple measures goes further than approaches used in many studies of acculturation and reproductive health, [13] the available measures still do not capture the complexity of the multidimensional nature of cultural adaptation. For example, these types of measures have been criticized due to their focus on the degree of adaptation to the "mainstream" culture, with a resulting 
neglect of the degree to which individuals maintain or separate from their original cultural identity as they adapt to the "mainstream" culture $[39,40]$. In addition to more detail about the acculturation of the subjects, it would be of interest to examine the subjects' parents' acculturation, as this can be hypothesized to impact teen sexual behavior. Therefore, we do not presume to have fully measured the effect of the cultural influences of countries of origin on teen birth. The direction in which this measurement error biases our results is difficult to estimate given the complex effect of measures of acculturation seen in our data, previous studies, and recent demographic data. Further, our measure of language was language spoken at home at the time of the interview among adult respondents, which is not necessarily reflective of the language spoken at home during their teenage years. This would likely lead to an over-estimate of the effect of language, as some English speaking adults may have been Spanish speaking during their teenage years.

Our measure of childhood socioeconomic status or position also has limitations. We only measured the respondents' highest level of parental education at one point in time, and this may not be equivalent to the parents' educational levels throughout the respondents' childhood. Further, while parental educational attainment is an important aspect of socioeconomic status, other relevant variables, such as income and wealth during a woman's childhood or teenage years, were not measured and are unlikely to be adequately reflected by education [41]. The effect of residual confounding by imperfect measurement of childhood socioeconomic factors would most likely be an underestimate of the effect on teen birth. An additional limitation is that our analysis is limited to women who gave birth, and therefore does not include the approximately $20 \%$ of women who never have a child [42] in the reference category of women who did not have a teenage birth. The effect of this on the measured associations cannot be determined. Finally, while our independent variablesmeasures of acculturation and respondents' parents' education-clearly precede our dependent variable of age at first birth, and a causal relationship is plausible, this crosssectional analysis cannot establish causality.

Despite these limitations, these findings suggest that socioeconomic factors may play an important role in teenage childbearing among Latinas. They raise the question whether the lack of addressing these issues, with perhaps too great a reliance on assumptions about the primacy of cultural factors, may have contributed to the slow progress in reducing teenage births among Latinas. If true, the policy implications are primarily outside the health sector, in the realm of social policies that influence children's socioeconomic status. The largest effect of the respondents' parents' education was seen among immigrant Latinas, predominantly from Mexico, whose parents were most likely educated outside of the United States; appropriate interventions for this group would consist of improving the educational opportunities and socioeconomic circumstances in the countries of origin. As the effect of parents' education among US-born Latinas, while smaller than among the immigrants, was still marked, this study suggests that increasing socioeconomic opportunities among Latinas, in this country and others, has the potential to decrease the persistent disparities in teenage childbearing between Latinas and non-Latina Whites.

Acknowledgment This publication was supported by NIH/NCRR/ OD UCSF-CTSI Grant Number KL2 RR024130. Its contents are solely the responsibility of the authors and do not necessarily represent the official views of the NIH.

Open Access This article is distributed under the terms of the Creative Commons Attribution Noncommercial License which permits any noncommercial use, distribution, and reproduction in any medium, provided the original author(s) and source are credited.

\section{References}

1. Chen, X. K., Wen, S. W., Fleming, N., Demissie, K., Rhoads, G. G., \& Walker, M. (2006). Teenage pregnancy and adverse birth outcomes: A large population based retrospective cohort study. International Journal of Epidemiology, 36, 368-373. doi:10.1093/ ije/dyl284.

2. Klepinger, D. H., Lundberg, S., \& Plotnick, R. D. (1995). Adolescent fertility and the educational attainment of young women. Family Planning Perspectives, 27, 23-28. doi:10.2307/2135973.

3. Hofferth, S., \& Hayes, C. (Eds.). (1987). Risking the future (Vol. 1, p. 138). Washington, DC: National Academy Press.

4. Moore, K. A., Myers, D. E., Morrison, D. R., Nord, C. W., Brown, B., \& Edmonston, B. (1993). Age at first childbirth and later poverty. Journal of Research on Adolescence, 3, 393-422. doi:10.1207/s15327795jra0304_5.

5. Stier, D. M., Leventhal, J. M., Berg, A. T., Johnson, L., \& Mezger, J. (1993). Are children born to young mothers at increased risk for maltreatment? Pediatrics, 91, 642-648.

6. Hubbs-Tait, L., Osofsky, J. D., Hann, D. M., \& Culp, A. M. (1994). Predicting behavior problems and social competence in children of adolescent mothers. Family Relations, 43, 439-446. doi: $10.2307 / 585376$.

7. Hardy, J. B., Shapiro, S., Astone, N. M., Miller, T. L., BrooksGunn, J., \& Hilton, S. C. (1997). Adolescent childbearing revisited: the age of inner-city mothers at delivery is a determinant of their children's self-sufficiency at age 27 to 33 . Pediatrics, 100, 802-809. doi:10.1542/peds.100.5.802.

8. Hamilton, B. E., Martin, J. A., \& Ventura, S. J. Births: Preliminary data for 2005. Available at http://www.cdc.gov/nchs/ data/hestat/prelimbirths05tables.pdf\#2. Accessed on December 22, 2008.

9. Centers for Disease Control Teen Pregnancy Fact Sheet. Available at http://www.cdc.gov/reproductivehealth/Adolescent ReproHealth/PDF/TeenPreg-FS.pdf. Accessed on November 30, 2008.

10. Vexler, E., \& Suellentrop, K. (2006). Bridging two worlds: How teen pregnancy prevention programs can better serve Latino 
youth. Washington, DC: National Campaign to Prevent Teen Pregnancy.

11. Driscoll, A. K., Brindis, C. D., Biggs, M. A., \& Valderrama, L. T. (2004). Priorities, progress and promise: A chartbook on Latino adolescent reproductive health. San Francisco, CA: University of California, San Francisco, Center for Reproductive Health Research and Policy, Department of Obstetrics, Gynecology and Reproductive Sciences and the Institute for Health Policy Studies.

12. Solorio, M. S., Yu, H., Brown, E. R., Becerra, L., \& Gelberg, L. (2004). A comparison of Hispanic and White adolescent females' use of family planning services in California. Perspectives on Sexual and Reproductive Health, 36, 157-161. doi:10.1363/361 5704.

13. Afable-Munsuz, A., \& Brindis, C. D. (2006). Acculturation and sexual and reproductive health of Latino youth in the United States: A literature review. Perspectives on Sexual and Reproductive Health, 38, 208-219. doi:10.1363/3820806.

14. Driscoll, A. K., Biggs, A. M., Brindis, C. D., \& Yankah, E. (2001). Adolescent Latino reproductive health: A review of the literature. Hispanic Journal of Behavioral Sciences, 23, 255-326. doi:10.1177/0739986301233001.

15. Frank, R., \& Heuveline, P. (2005). A crossover in Mexican and Mexican-American fertility rates: Evidence and explanations for an emerging paradox. Demographic Research, 12, 77-104.

16. Russell, S. T., \& Lee, F. C. H. (2004). Practitioners' perspectives on effective practices for Hispanic teenage pregnancy prevention. Perspectives on Sexual and Reproductive Health, 36, 142-149. doi:10.1363/3614204.

17. Gilliam, M. L., Berlin, A., Kozloski, M., Hernandez, M., \& Grundy, M. (2007). Interpersonal and personal factors influencing sexual debut among Mexican-American young women in the United States. The Journal of Adolescent Health, 41, 495-503. doi:10.1016/j.jadohealth.2007.05.009.

18. Hunt, L. M., Schneider, S., \& Comer, B. (2004). Should "acculturation" be a variable in health research? A critical review of research on US Hispanics. Social Science and Medicine, 59, 973-986. doi:10.1016/j.socscimed.2003.12.009.

19. Frost, J. J., \& Driscoll, A. K. (2006). Sexual and reproductive health of U.S. Latinas: A literature review. Occasional Report. New York: Guttmacher Institute, No. 19.

20. Santelli, J. S., Lowry, R., Brener, N. D., \& Robin, L. (2000). The association of sexual behaviors with socioeconomic status, family structure, and race/ethnicity among US adolescents. American Journal of Public Health, 90, 1582-1588. doi:10.2105/AJPH.90. 10.1582 .

21. Ramirez-Valles, J., Zimmerman, M. A., \& Juarez, L. (2002). Gender differences of neighborhood and social control process: a study of the timing of first intercourse among low-achieving, urban, African American youth. Youth \& Society, 33, 418-441. doi:10.1177/0044118X02033003004.

22. Rose, A., Koo, H. P., Bhaskar, B., Anderson, K., White, G., \& Jenkins, R. R. (2005). The influence of primary caregivers on the sexual behavior of early adolescents. The Journal of Adolescent Health, 37, 135-144. doi:10.1016/j.jadohealth.2005.02.009.

23. Carvajal, S. C., Parcel, G. S., Banspach, S. W., Basen-Engquist, K., Coyle, K. K., Kirby, D., et al. (1999). Psychosocial predictors of delay of first sexual intercourse by adolescents. Health Psychology, 18, 443-452. doi:10.1037/0278-6133.18.5.443.

24. Hayward, M. D., Grady, W. R., \& Bill, J. O. G. (1992). The influence of socioeconomic status on adolescent pregnancy. Social Science Quarterly, 73, 750-772.

25. Upchurch, D. M., Astone, N. M., \& McCarthy, J. (1990). Influences of family background on adolescent childbearing: from the 1940s to the 1980s. Paper presented at: The annual meeting of the Population Association of America, May 3-5, Toronto, Canada.
26. Hogan, D. P., \& Kitagawa, E. M. (1985). The impact of social status, family structure, and neighborhood on the fertility of Black Adolescents. American Journal of Sociology, 90, 825-855. doi:10.1086/228146.

27. Xie, H., Cairns, B. D., \& Cairns, R. B. (2001). Predicting teen motherhood and teen fatherhood: Individual characteristics and peer affiliations. Social Development, 10, 488-511. doi: 10.1111/1467-9507.00177.

28. U.S. Census Bureau. (2005). Available at http://www.census.gov/ Press-Release/www/releases/archives/04eductableA.xls. Accessed on November 10, 2007.

29. US Census Bureau. (2006). Available at http://www.census.gov/ hhes/www/poverty/poverty06/table3.pdf. Accessed on November 10, 2007.

30. Gibson, J. W., \& Lanz, J. B. (1991). Factors associated with Hispanic teenagers' attitude toward the importance of birth control. Child and Adolescent Social Work Journal, 8, 399-415. doi:10.1007/BF00758163.

31. Adolph, C., Ramos, D. E., Linton, K. L., \& Grimes, D. A. (1995). Pregnancy among Hispanic teenagers: Is good parental communication a deterrent. Contraception, 51, 303-306. doi:10.1016/ 0010-7824(95)00081-K.

32. Driscoll, A. K., Sugland, B. W., Manlove, J., \& Papillo, A. R. (2005). Community opportunity, perceptions of opportunity, and the odds of an adolescent birth. Youth \& Society, 37, 33-61. doi: 10.1177/0044118X04267493.

33. Denner, J., Kirby, D., Coyle, K., \& Brindis, C. (2001). The protective role of social capital and cultural norms in Latino communities: A study of adolescent births. Hispanic Journal of Behavioral Sciences, 23, 3-21. doi:10.1177/0739986301231001.

34. Kirby, D., Coyle, K., \& Gould, J. B. (2001). Manifestations of poverty and birthrates among young teenagers in California zip code areas. Family Planning Perspectives, 33, 63-69. doi: $10.2307 / 2673751$.

35. Fisher, T. D. (1990). Characteristics of mothers and fathers who talk to their adolescent children about sexuality. Journal of Psychology \& Human Sexuality, 3, 53-70. doi:10.1300/J056 v03n02_05.

36. Duncan, G. J. (1994). Families and neighbors as sources of disadvantage in the schooling decisions of Black and White adolescents. American Journal of Education, 103, 20-53. doi: $10.1086 / 444088$.

37. Stanton-Salazar, R. D., \& Dornbusch, S. M. (1995). Social capital and the reproduction of inequality: Information networks among Mexican-origin high school students. Sociology of Education, 68, 116-135. doi:10.2307/2112778.

38. Dyk, P. K. (2004). Complexity of family life among the low income and working poor: Introduction to the special issue. Family Relations, 53, 122-126. doi:10.1111/j.0022-2445.2004.00002.x.

39. Phinney, J. S. (1990). Ethnic identity in adolescents and adults: Review of research. Psychological Bulletin, 108, 499-514. doi: 10.1037/0033-2909.108.3.499.

40. Abraido-Lanza, A. F., Armbrister, A. N., Florez, K. R., \& Aguirre, A. N. (2006). Toward a theory-driven model of acculturation in public health research. American Journal of Public Health, 96, 1342-1346. doi:10.2105/AJPH.2005.064980.

41. Braveman, P. A., Cubbin, C., Egerter, S., Chideya, S., et al. (2005). Socioeconomic status in health research: One size does not fit all. Journal of the American Medical Association, 294, 2879-2888. doi:10.1001/jama.294.22.2879.

42. Dye, J. L. (2005). Fertility of American women: June 2004. Current Population Reports, P20-555. U.S. Census Bureau, Washington, DC. 